\title{
Robustness and Accuracy Test of Particular Matter Prediction Based on Neural Networks
}

\author{
Jiamei Deng ${ }^{1}$, Shaohua Zhong ${ }^{2}$, Andrew Ordys ${ }^{1}$ \\ ${ }^{1}$ School of Mechanical and Automotive Eng., Kingston University London, UK SW15 3DW \\ ${ }^{2}$ School of Automotive Engineering, Wuhan University of Technology, Wuhan 430070, China \\ Email: j.deng@kingston.ac.uk, szhong@whut.edu.cn,
}

Received 2013

\begin{abstract}
The increasingly stringent emissions regulations require that engine manufacturers must reduce emissions of particulate matter (PM). PM is made up mainly of carbon in the form of PM and complex compound that are absorbed into the PM particles. Significant quantities PM poses a threat to health. Technologies available for PM reduction are heavily dependent on after-treatment systems, which are respectively active and passive. An active trap system requires a control unit to trigger and control the regeneration process (clean the trap system). The measurement of PM is crucial for the trap system to enable the fine judgment as to when to initiate the process. If the regeneration is too infrequent, the filter will block. The frequency of the regeneration and the life of the filter are compromised. Diesel engine particular matter prediction has always been a major challenge to the industry. A simple way to handle the PM estimation is to use black-box modelling as described in [J. Deng, B. Maass, R. Stobart, PM prediction in both steady state and transient operation of diesel engines, Proc IMechE, Part D: Journal of Automobile Engineering, 2011, 225, in press, DOI: 10.1177/0954407011418029]. This method is used to estimate the PM successfully in both steady and transient engine operation condition. The main question is how robust and accurate the neural networks are for a regeneration trigger signal of diesel particular filters. In order to answer such a question, the robust test of PM estimation is carried out based on different composition of bio-diesel. In this paper, regular diesel fuel will be blended with up to $20 \%$ bio-diesel to test the effect of different fuel resources on particulate matter. Bio-diesel is often added to regular diesel fuel to improve the burning properties and reduce carbon emissions, also is alternative source of fuels. The aim of this paper with these tests is to ensure that with a realistic change in the fuel composition the estimation of PM is still accurate. Therefore, neural networks could be used to produce a regeneration signal for diesel particular filter. In this paper a virtual sensor is proposed to measure the PM. The purpose of the proposed virtual sensor is to estimate the accumulation of PM and to trigger a regeneration cycle. The virtual sensor based on neural network is used to estimate the PM. In this paper, the performance, robustness and accuracy of simulated the sensor are evaluated in measuring the particulate amount for non-road transient cycle tests.
\end{abstract}

Keywords: Neural Networks; Particulate Matter; Smoke; Prediction; Robustness

\section{Introduction}

The increasingly stringent emissions regulations require that engine manufacturers must reduce emissions of particulate matter (PM). PM is made up mainly of carbon in the form of PM and complex compound that are absorbed into the PM particles. Significant quantities of PM pose a threat to health. Technologies available for PM reduction are heavily dependent on after-treatment systems, which are respectively active and passive. Diesel Particulate Filters (DPFs) are kinds of after-treatment system and can effectively reduce the level of PM emissions to ambient background levels [1].

If PM accumulates on the DPF, the large amount of heat release during regeneration cannot effectively be dissipated. This could result in filter damage, for example, the formation of cracks or regions which may be locally melted. Extensive studies try to identify all mechanisms related to DPF failures and to develop strategies to avoid these failures [4]. On the other hand, if the DPF regenerates too frequently, it will also cause additional fuel penalties. Therefore, accurate knowledge of the DPF PM loading state is important for improving fuel economy and extending DPF service life. It is also very critical for the upcoming on-board diagnostics regulations [5].

A normal DPF system is very expensive (a heavy-duty catalyzed DPF unit costs about \$5000) and a special design one will cost much more. Therefore, controlling the 
DPF regeneration safely is highly critical [4]. The timing for safely and efficiently regenerating the DPF has become a major industrial challenge.

It can be seen that a control unit to trigger and control the regeneration process is crucial for both DPF life and fuel economy. The measurement of PM is crucial for the trap system to enable the fine judgment as to when to initiate the process. The frequency of the regeneration and the life of the filter are compromised.

Diesel engine PM Prediction has always been a major challenge to the industry $[6,7,8]$. The conventional method to estimate the PM loading is pressure drop measurements. But it is affected by exhaust flow variations and exhibits a low degree of sensitivity to DPF PM loading and has bad dynamic response over transient operating conditions. There is study investigating the use of radio frequency (RF) to directly monitor measure DPF PM accumulation levels. However, this technique is not mature enough to be applied in commercial applications as it is not easy to be calibrated. Hence, more reliable, stable and accurate PM loading estimation or sensing method should be studied. Computational fluid dynamics (CFD) based PM models are computationally intensive and are not suitable for control purpose and real time measurement. Recently, neural networks have been used in a wide variety of automotive applications. The advantage of neural networks is their ability to be used as an arbitrary function approximation mechanism which has no requirement to represent the complex underlying process and is an economic way to obtain the measurement. Model based PM emission prediction method could be a good alternative way [9]. Neural networks have been successfully used for emissions prediction [10]. He et al. [11] built a model that considers several engine parameters such as boost pressure and exhaust gas recirculation (EGR) and it generates several outputs including $\mathrm{PM}$ emissions. Maass et al. presented a smoke prediction neural network model using a three-layer autoregressive model with exogenous inputs (NLARX) model to predict PM [12]. Bose and Kumar [13] use fuzzy logic to predict the engine emissions.

The simple way to handle the PM estimation is to use black-box modeling as described in [14].This method is used to estimate the PM successfully in both steady and transient engine operation condition. The disadvantage is the robustness and accuracy of the PM prediction based on neural networks while different fuels are used in the engines. The neural network model is built on training data, which has no information on fuel types. Therefore, the test of robustness and accuracy is important for the PM estimation based on neural networks. In order to carry out the robust and accurate tests, different compositions of Bio-diesel with standard diesel EN590 are used.
In order to improve the engine performance and emissions and ensure the sustainability of the fuel supplies, bio-diesel is a promising alternative for diesels. Biodiesel leads to significant reductions in PM emissions, although the percentage reduction varied with fuel composition and engine technology. The average PM reductions are $26 \%$ compared to conventional diesel fuel [15].

In this paper, standard diesel fuel (EN590) will be blended with up to $20 \%$ bio-diesel fuel to test the effect of different fuel resources on PM. The aim with these tests is to ensure that with a realistic change in the fuel composition the estimation of PM based on neural networks is still accurate enough to trig a regeneration cycle. The virtual sensor of PM measurement based on neural networks will be used to estimate the PM. With the simulated sensor we will evaluate its performance, robustness and accuracy in predicting the PM.

In this paper a virtual sensor is proposed to measure the PM. The purpose of the proposed virtual sensor is to estimate the PM and to trigger a regeneration cycle for diesel particulate filter.

In Section 1 - introduction, a brief background of the research is introduced. Section 2 reviews the non-linear autoregressive model with exogenous inputs (NLARX) neural networks that can be used to predict PM. Section $\mathbf{3}$ described details of the test facility. Section $\mathbf{4}$ describes the data collection and neural network training and robustness test. Section 5 provides conclusions on this work.

\section{Neural Networks}

The field of virtual sensing has become more and more popular with growing systems complexity such as in combustion engine control. Its origin lies in the field of estimators which are specified through physical and numerical relations whereas virtual sensors are characterized through black-box approaches such as neural networks.

Neural networks can be split into the following three categories:

1) single-layer feed forward networks (SLFN),

2) multi-layer feed forward networks (MLFN),

3) recurrent neural network (RNN).

The chosen network structure or architecture is crucial for the output performance. Depending on the systems characteristic: linear or non-linear, static or dynamic, the network needs to be designed accordingly. Here, the prediction of PM is recognised as highly dynamic and non-linear that implies a recurrent network structure has to be chosen to offer sufficient predictive capability. The NLARX structure can accommodate the dynamics of the system by feeding previous network outputs back into the input layer. It also enables the user to define how many 
previous output and input time steps are required for representing the systems dynamics best. In this paper a NLARX model is applied as it is suitable for non-linearity of the problem. Although an important result of approximation theory is that a three-layer feed-forward neural network with sigmoid activation functions in the hidden layer and linear activation functions in the output layer has the ability to approximate any continuous mapping to arbitrary precision, provided that the number of units in the hidden layer is sufficiently large [16]. However, the performance of feed-forward neural networks is limited due to limitations to the number of units in the hidden states. Performance is further limited by the memory of personal computers. It is for this reason that, SLFN and MLFN have not formed part of the work reported in this paper.

A typical structure of an NLARX model is illustrated in Figure 1. The inputs are represented by $u(n)$ and the outputs are described by $\mathrm{y}(\mathrm{n})$. The formulation of this NLARX model can be described as:

$$
y(n)=F(y(n-1), \ldots, y(n-n y), u(n), \ldots, u(n-n u+1))
$$

where ny is number of past output terms used to predict the current output, $n u$ is number of input terms used to predict the current output.

Each output of an NLARX model is a function of regressors which are transformations of past inputs and past outputs. Usually this function has a linear block and a nonlinear block. The model output is the sum of the outputs of the two blocks. Typical regressors are simply delayed input or output variables. More advanced regressors are in the form of arbitrary user-defined functions of delayed input and output variables.

NLARX model training can be cast as a non-linear unconstrained optimization problem:

$$
\min _{\theta} F_{M}\left(\theta, Z_{M}\right)=\frac{1}{2 M} \sum_{k=1}^{M}\|y(k)-\hat{y}(k \mid \theta)\|^{2}
$$

where $Z_{M}=[y(k), u(k)]_{k=1, \ldots, M}$ is a training data set, $\mathrm{y}(\mathrm{k})$ represents the measured output which is the measured PM in the training set, $\hat{\mathrm{y}}(\mathrm{k} \mid \theta)$ is the NLARX output which is predicted PM, $\|\|$.2 is 2-norm operation, $\theta$ is a parameter vector, where $\theta=\left[\theta_{1}, \cdots, \theta_{i}, \cdots, \theta_{p}\right]$ and $\mathrm{p}$ is the number of parameters.

The training process is described as follows. Given a neural network described by Equation (1), there is an error metric, that we refer to as performance index of Equation (2), which is to be minimized. This index is a representation of the approximation of the network to some given training patterns. The task will be to modify the network parameters $\theta$ to reduce the index $F_{M}\left(\theta, Z_{M}\right)$ over the complete trajectory to achieve the minimal value. In this paper the neural networks are trained using gradient descent algorithms while the initial value of $\theta$ is perturbed several times in order to avoid the local minimal solution. The gradient descent methods will calculate the vector $\nabla_{\theta} \mathrm{F}_{\mathrm{M}}$ whose elements are $\frac{\delta \mathrm{F}_{i}}{\delta \theta_{i}} \quad(i=1, \cdots, i, \cdots, p)$. The training algorithm will find the parameters of the network for which the performance index has reached a desirable value. Given a vectorising trajectory for the network output and training patterns, the performance index is the Euclidean norm of the error matrix of the whole training batch for the output PM.

This model has predicted PM accurately with R-square $=0.99$ as shown in [14]. The inputs for this model are torque, speed, the first deritatives of torque and speed, and the second deritative of torque. This paper would like to investigate how robust the PM sensors are and whether it is suitable to give an alarm signal to trig the regeneration cycle for the DPF when different composition of fuels are used.

NLARX could be used to predict the PM of diesel engines and thus produce a trigger signal for the regeneration cycles of DPFs. This is a virtual sensor (software) that takes account of various engine parameters to calculate particulate matters.

The advantage of neural networks is their ability to be used as an arbitrary function approximation mechanism which has no requirement to represent the complex underlying process and is an economic way to obtain the measurement. A potential disadvantage of the neural network is that how robust and accurate it is for the prediction of PM when different fuels are used on engines. In order to answer these questions, bio-diesel blended with EN590 will be used for the robust and accurate test of neural networks bio-diesel is used as an alternative diesel has become more attractive due to its low emissions properties.

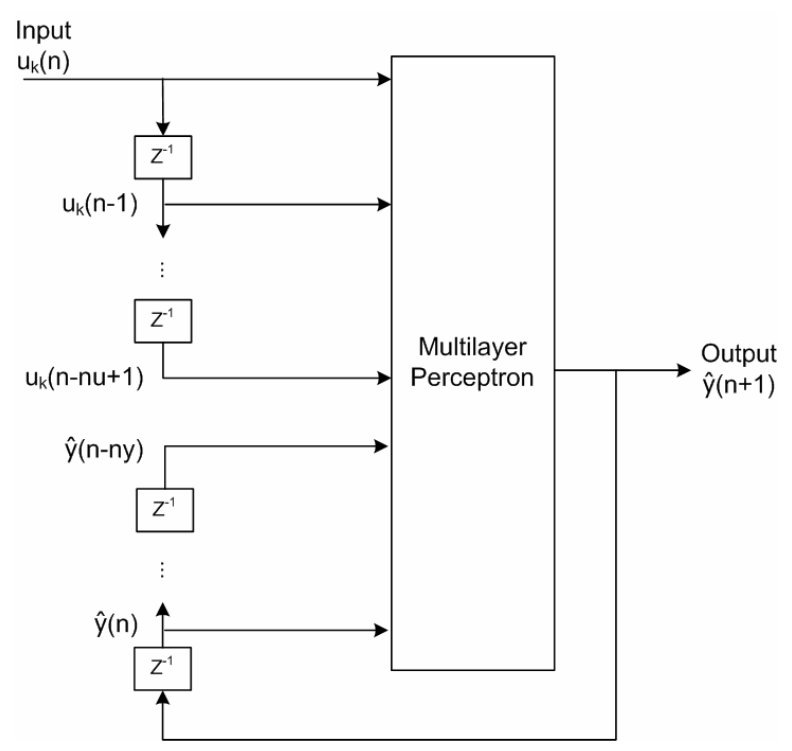

Figure 1. NLARX canonical structure. 


\section{Test Facility}

The engine employed in this study is a Peugeot $2.0 \mathrm{~L}$ HDI Engine. This is a 4-cylinder engine with a Bosch common rail fuel system and turbo-charger. The engine calibration used in this work produces a peak torque of $149 \mathrm{Nm}$ at $2002 \mathrm{rpm}$.

The engine is fully instrumented to measure air, fuel and cooling system pressures, temperatures and flow rates. Emissions data is gathered principally using an AVL 415 smoke and 439 opacity meters (offering steady state and transient measurement respectively) and a Horiba Horiba MEXA 9100 exhaust gas analyser measuring nitrous oxide, carbon dioxide, carbon monoxide, unburnt hydrocarbons and oxygen..

Figure 2 shows the engine facility. The engine is situated in the lab of the Mechanical and Automotive Eng of Kingston University. An AVL 439 opacity meter is integrated into the engine exhaust and provides a fast measurement of the exhaust particulate concentration. This instrument is highly suited to the study of engine speed-torque transient events during which the control of exhaust PM is difficult; events such as these contribute significantly to the total PM produced by the engine. Accurately predicting the particulates produced during these events is essential for any model. This is particularly so for a model which has the potential to be deployed as a virtual sensor for determining the optimum point in time for diesel predicate filter regeneration.

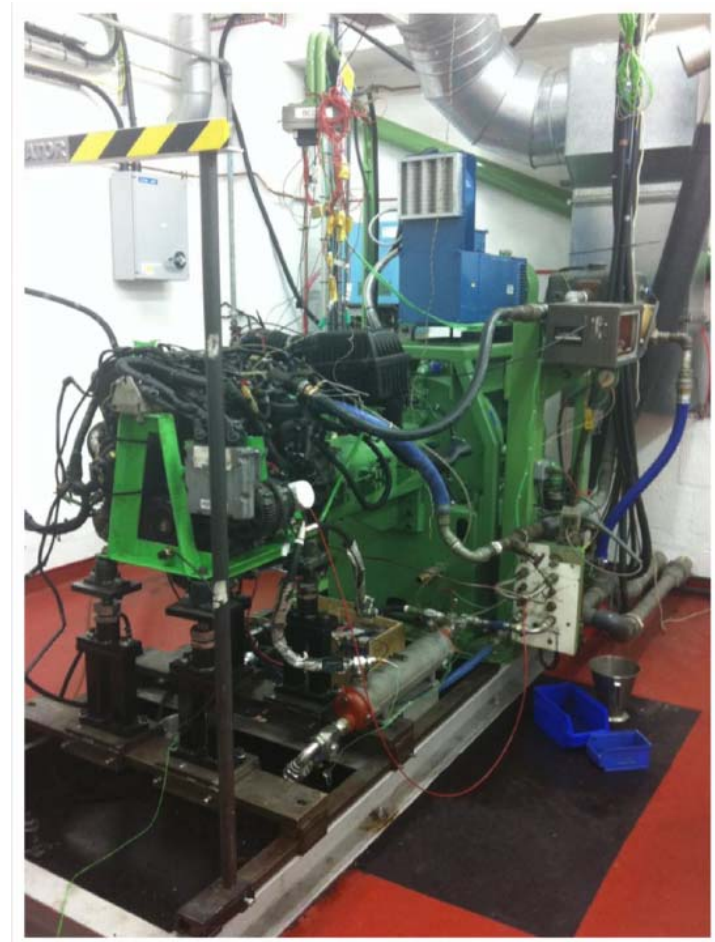

Figure 2. Peugeot 2.0-litre diesel engine and particulate measurement instruments.

\section{Data Collection}

All the engine parameters are recorded in a $100 \mathrm{HZ}$ sampling frequency under the test conditions. For an initial model set-up NRTC (Nonroad Transient Cycle) are operated on a Peugeot 2.0 L HDI Engine at the test facilities shown in Figure 1. The ECM is set to standard calibration mode. Peugeot 2.0 L HDI Engine runs on a transient NRTC in order to catch as much as dynamics of the engine.

NRTC is an engine dynamometer transient driving schedule of total duration of about 1200 seconds. The speed and torque during the NRTC test is shown in Figure 3 and Figure 4. Motivation for this choice of cycle is twofold. First, experience has shown that this is one of the most challenging cycles in terms of emissions modelling. Secondly, meeting emission formation requirements under the NRTC cycle is also a major concern to engine manufacturers. The current trend is to design engines which are marginally passing legislative emission test, thereby the use of reliable and highly accurate emissions models is of critical importance.

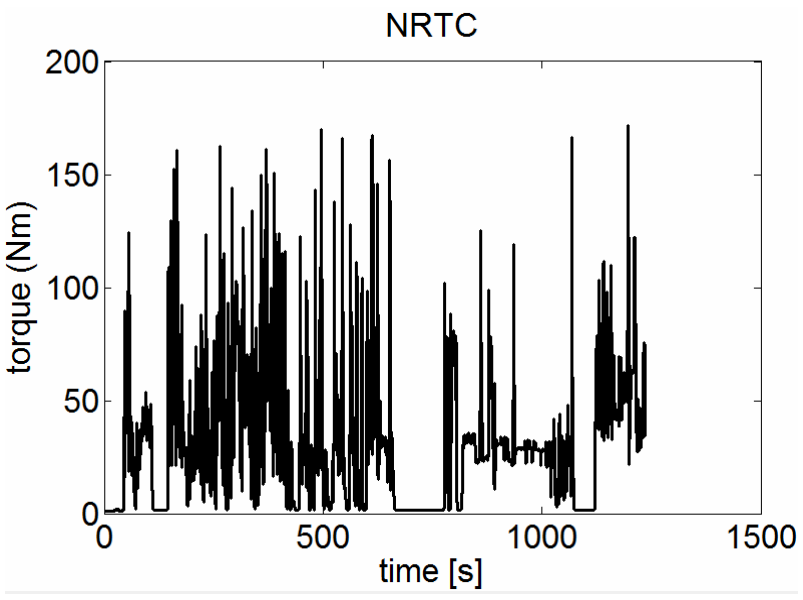

Figure 3. The torque information of the NRTC.

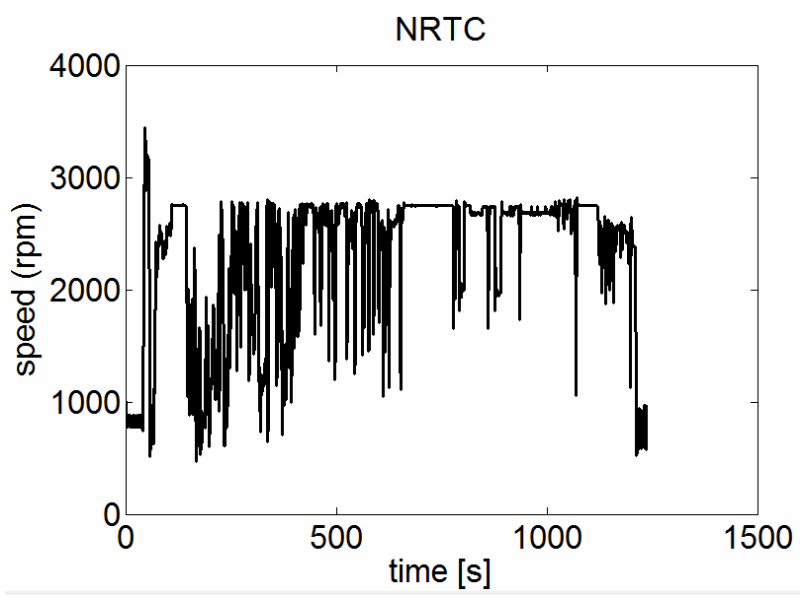

Figure 4. The speed information of the NRTC. 
The test is completed with $70 \%$ maximum load and full speed range covering a wide range of engine transients in different frequencies and combinations. The test facility is only allowed to run NRTC at $70 \%$ load, also there are some points that the torque drops and this problem cannot be solved in the current engine setting-up. Figure 5 the PM results of NRTC

In order to test the robustness of the PM virtual sensor based on neural network model. Different bio-diesel blends mixed with standard diesel have been tested for PM in the engine test cell shown in Figure 2. The blend is made up of the standard diesel fuel (EN590) and different portion of bio-diesel fuel in volume in the experiment. Different fuel blends which are made of up to $20 \%$ biodiesel-diesel will be used to test the effects of different fuel sources on PM emission. The test results are shown in the Figure 5. It can be seen that the PM with different percentages of bio-diesel will be different. Therefore it is necessary to test whether the PM prediction based on neural networks is robust enough to make a PM estimation for the different bio-diesel blends.

The test results of the standard diesel and the blend of the standard diesel and 20\% bio-diesel have been shown in Figure 6. It can be seen clearly that the standard diesel produce higher PM emissions than that of $20 \%$ bio-diesel blend with the same engine and without modification with hardware and software.

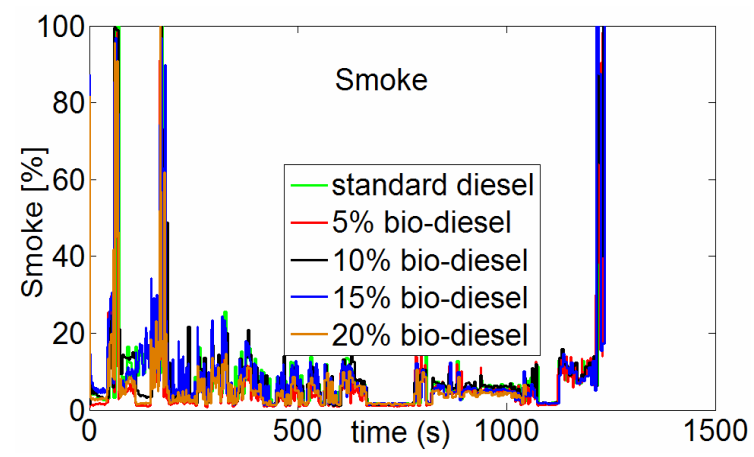

Figure 5. Comparisons of the result for different percentage bio-diesel and diesel.

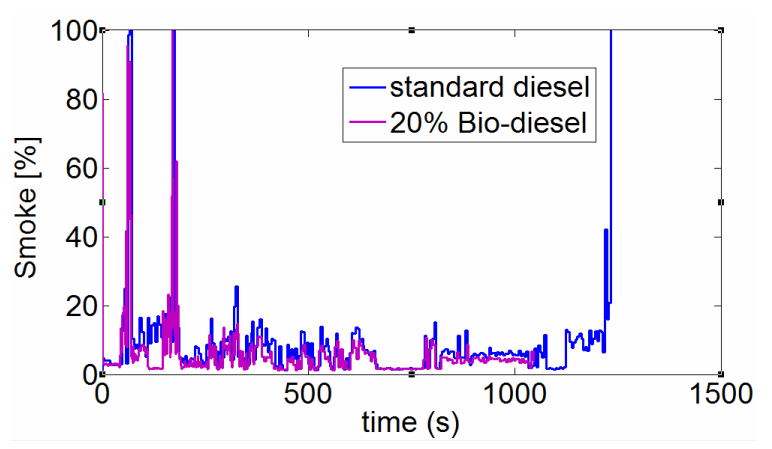

Figure 6. Comparison of opacity emission between standard fuel and $20 \%$ bio-diesel during NRTC.

\section{Robustness Test of PM Sensors}

The PM virtual sensor which is an NLARX model identified from engine test data is implemented [14] and tested against data that collected for the blends of different percentages of bio-diesel and standard diesel. In order to test the robustness of the virtual sensor, the data of the blend of $10 \%$ bio-diesel and standard diesel is used to train the neural networks. 160 seconds of NRTC data between 240 - 400 seconds will be enough to train a neural network [17]. The data between 240 and 400 seconds data for training purpose is used. The rest of NRTC data will be used for validation purpose. Inputs for training the neural network using torque, speed and their derivatives as inputs.

The resulting NLARX model achieves a result of Rtrain $^{2}=0.99$ and Rvalid ${ }^{2}=0.99$ as shown in Figure 7. This result shows that even if other data, uncorrelated to the used training data can be predicted to a highly sufficient correlation standard. The inputs are torque, speed and its derivatives. They provide sufficient feature detail for the network to generalise unseen and uncorrelated features.

The PM model based on neural networks has been obtained by using the $10 \%$ bio-diesel testing data. The estimation of PM based on this model is very accurate for $5 \%$ bio-diesel blend $\left(\right.$ Rpredict $\left.^{2}=0.99\right), 10 \%$ bio-diesel blend $\left(\right.$ Rpredict $\left.^{2}=0.99\right), 15 \%$ bio-diesel blend $\left(\right.$ Rpredict $^{2}$ $=0.99), 20 \%$ bio-diesel blend $\left(\right.$ Rpredict $\left.^{2}=0.99\right)$. This accuracy can also be seen from the prediction figures in Figure 7, Figure 9, Figure 10, Figure 11, which show the PM comparison between the neural network model and NRTC cycle tests for different percentage blends. But it cannot predict the standard diesel $\left(\right.$ Rpredict $\left.^{2}=0.66\right)$ accurately, shown in Figure 8. It can be concluded that PM models based on neural networks, which are trained based on $10 \%$ bio-diesel, are quite robust to predict the emissions for the blends of different percentage bio-diesels and standard diesel. However, the prediction for the PM of the standard diesel is not accurate.

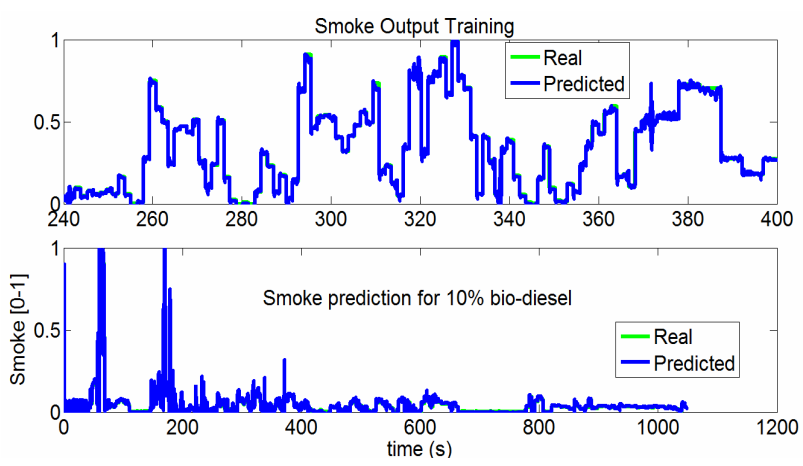

Figure 7. NLARX correlation results for training with 160 seconds data and validation agains residual cycle data Network Topology: $y(n+1)=F(y(n), \ldots, y(n-4), u(n-1))$. 


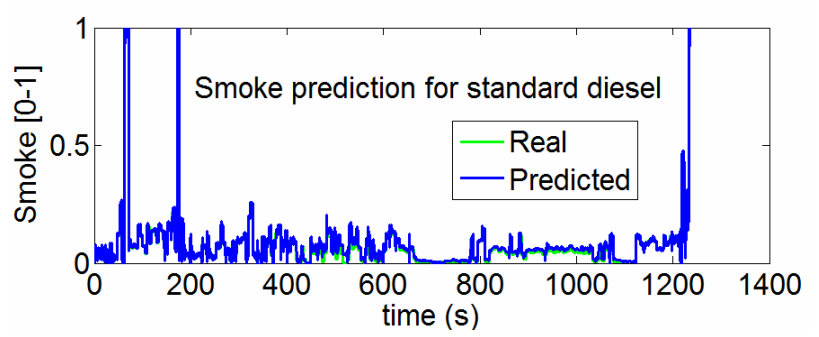

Figure 8. PM measured by opacity meter and estimated by NN model for Standard diesel EN590.

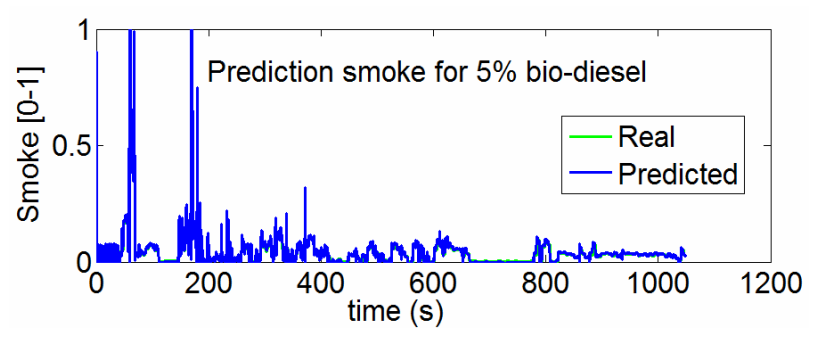

Figure 9. PM measured by opacity meter and estimated by NN model for 95\% EN590 and 5\% bio-diesel.

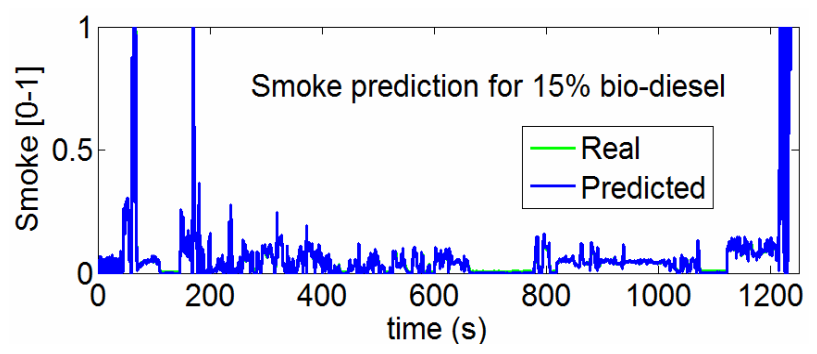

Figure 10. PM measured by opacity meter and estimated by NN model for $85 \%$ EN590 and 15\% bio-diesel.

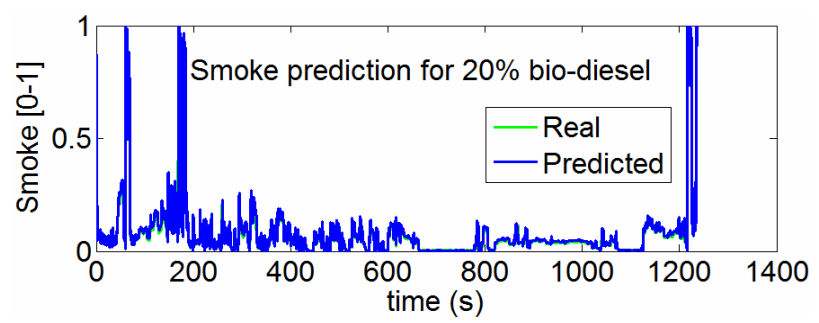

Figure 11. PM measured by opacity meter and estimated by NN model for $80 \%$ EN590 and $20 \%$ bio-diesel.

\section{Conclusions and Discussion}

Neural network model has been used to predict the PM accurately for the particular blended bio-diesel. For other percentage blended bio-diesel, the neural networks could still predict PM accurately. But the prediction is not accurate for the PM of the standard diesel based on $10 \%$ bio-diesel data. For the tests, it could be seen that the blend of bio-diesel with the standard diesel fuel could reduce PM emission without any engine modification. The PM model based on neural networks which is trained by one of the $10 \%$ bio-diesel NRTC transient test is good for PM estimation with other percentages of bio-diesel, but has big error with EN590 standard diesel fuel in this paper.

\section{Acknowledgements}

The authors would like to thank the financial support of Royal Academy of Engineering for the research exchange scheme with China.

\section{REFERENCES}

[1] A. P. E. York, M. Ahmadinejad, T. C. Watling, A. P. Walker, J. P. Cox, J. Gast, P. G. Blakeman and R. Allansson, "Modeling of the Catalyzed Continuously Regenerating DPF (CCR-DPF) System: Model Development and Passive Regeneration Studies," SAE 200701-0043.

[2] Allansson, R. Cooper, J. Barry, J. E. Thoss, A. Uusimäki, A. P. Walker and J. P. Warren, "European Experience of High Mileage Durability of Continuously Regenerating Diesel Particulate Filter Technology," SAE 200001-0480.

[3] R. Allansson, P. G. Blakeman, B. J. Cooper, H. Hess, P. J. Silcock and A. P. Walker, "Optimising the Low Temperature Performance and Regeneration Efficiency of the Continuously Regenerating Diesel Particulate Filter (CR-DPF) System," SAE 2002-01-0428.

[4] R. Zhan, Y. Huang and M. Khair, "Methodologies to Control DPF Uncontrolled Regenerations," SAE 2006-01-1090.

[5] M. P. Sturgess, S. F. Benjamin and C. A. Roberts, "Spatial Conversion Profiles within an SCR in a Test Exhaust System with Injection of Ammonia Gas Modelled in CFD Using the Porous Medium Approach," SAE 2010-01-2126.

[6] Y. Liu, "Diesel Engine Modelling and Optimization for Emission Reduction," PhD Thesis, University of Wisconsin-Madison, 2005.

[7] I. M. Kennedy, "Models of PM Formation and Oxidation," Progress in Energy and Combustion Science, Vol. 23, No. 2, 1997, pp. 95-132. doi:10.1016/S0360-1285(97)00007-5

[8] H. P. M. Bockhorn, "Formation in Combustion: Mechanism and Models," Springer-verlag, Heidelberg, Vol. 59, 1994. doi:10.1007/978-3-642-85167-4

[9] A. Westlund, "Measuring and Predicting Transient Diesel Engine Emissions," Academic Thesis, Royal Institute of Technology, Stockholm, 2009.

[10] B. Wu, R. G. Prucka, Z. S. Filipi, D. M. Kramer and G. L. Ohl, "Cam-Phasing Optimization Using Artificial Neural Networks as Surrogate Models-Fuel Consumption and NOx Emissions," SAE 2006-01-1512.

[11] Y. He and C. J. Rutland, "Application of Artificial Neural Networks in Engine Modeling," International Journal of Engine Research, 2004, Vol. 5, No. 4, pp. 281-296. 
doi: $10.1243 / 146808704323224204$

[12] B. Maass, R. Stobart and J. Deng, "Diesel Engine Emissions Prediction with Parallel Neural Networks," American Control Conference, 2009.

[13] N. Bose and N. S. Kumar, "Prediction of Engine Emissions through Fuzzy Logic Modelling," International Conference on Computational Intelligence and Multimedia Applications, 2007.

[14] J. Deng, B. Maass and R. Stobart, "PM Prediction in Both Steady State and Transient Operation of Diesel Engines," Journal of Automobile Engineering, Vol. 226, No. 2, 2011, pp. 260-274. doi:10.1177/0954407011418029
[15] T. L. Alleman and R. L. McCormick, "Fischer-Tropsch Diesel Fuels - Properties and Exhaust Emissions: A Literature Review," SAE 2003-01-0763.

[16] K. Funahashi, "On the Approximate Realization of Continuous Mappings by Neural Networks," Neural Networks, Vol. 2, No. 3, 1989, pp. 183-192. doi: /10.1016/0893-6080(89)90003-8

[17] J. Deng, M. Bastian and R. Stobart, "Minimum Data Requirement for Neural Networks Based on Power Density Analysis," IEEE Transactions on Neural Networks, Vol. 23, No. 4, 2012, pp. 585-595. doi:10.1109/tnnls.2012.2183887. 